Document downloaded from:

http://hdl.handle.net/10251/120667

This paper must be cited as:

Rodríguez-Sánchez, MDLA.; Cuenca, L.; Ortiz Bas, A. (2018). FIWARE Open Source Standard Platform in Smart Farming - A Review. IFIP Advances in Information and Communication Technology. 534:581-589. https://doi.org/10.1007/978-3-319-99127-6_50

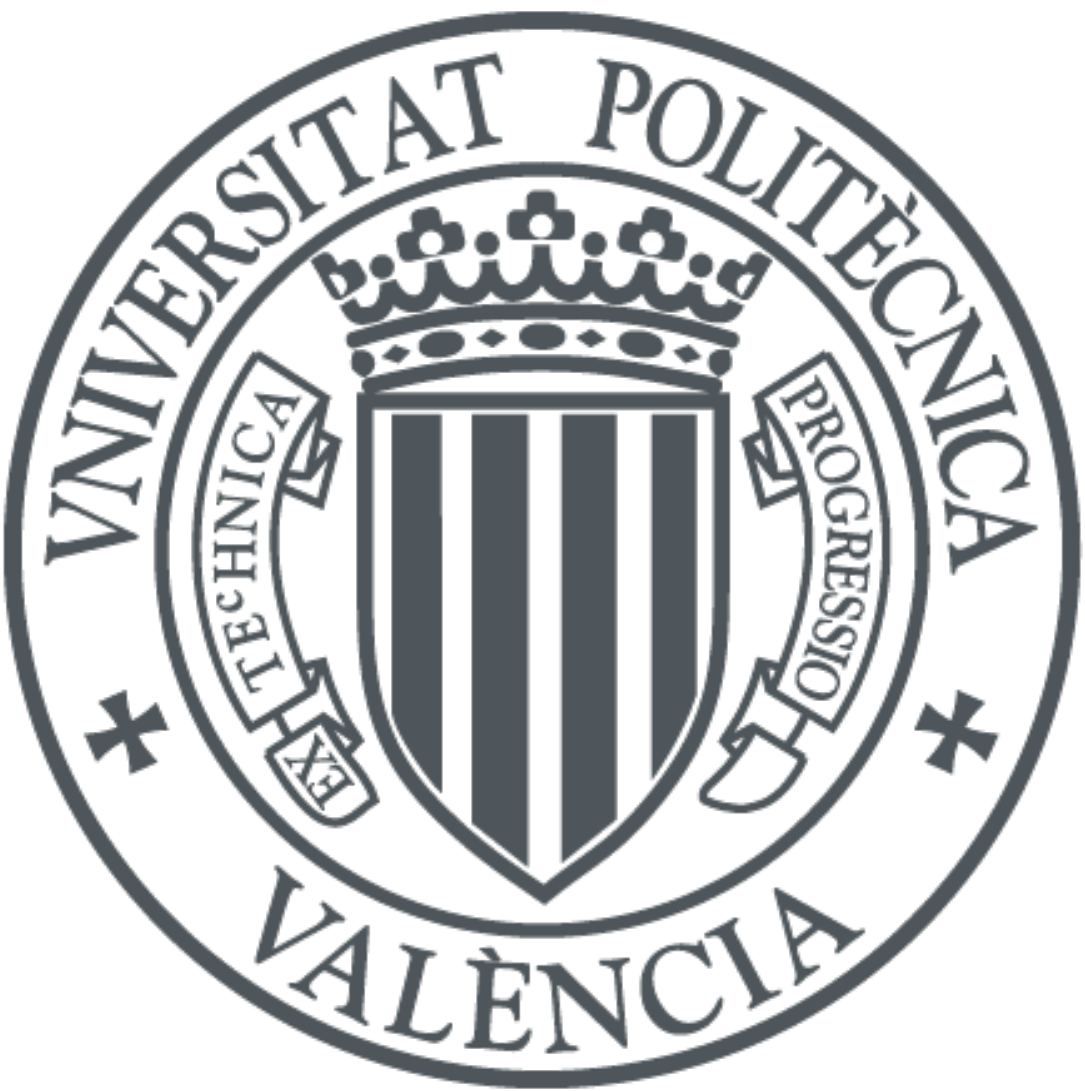

The final publication is available at

http://doi.org/10.1007/978-3-319-99127-6_50

Copyright Springer

Additional Information 


\title{
FIWARE Open Source Standard Platform in Smart Farming - A Review
}

\author{
Maria Angeles Rodriguez ${ }^{1}$, Llanos Cuenca ${ }^{2(\bowtie)}$, and Angel Ortiz ${ }^{2}$ \\ ${ }^{1}$ School of Informatics, Universitat Politècnica de València, \\ Camino de Vera S/N, 46002 València, Spain \\ marodsa4@inf.upv.es \\ 2 Research Centre on Production Management and Engineering (CIGIP), \\ Universitat Politècnica de València, Camino de Vera S/N, 46002 València, Spain \\ \{llcuenca, aortiz\}@cigip.upv.es
}

\begin{abstract}
FIWARE is an open source platform for the deployment of Internet of Things (IoT) applications, driven by European Union and managed by FIWARE Foundation. Recently, FIWARE Foundation has launched his new product Agricolus, which focus on Smart Farming and it uses FIWARE infrastructure. Agricolus manages to bring Hardware and Software together in a decision-making process that support farming activities and offers a "plug and play" interface for precision agriculture. This is encompassed by the phenomenon of Smart Farming, which is a development that take advantage of the use of Information Communication Technologies (ICT) in the daily farm management. This review aims to gain insight into the state-of-the-art of FIWARE in Smart Farming and identify the components of Agricolus in comparison with essential FIWARE architecture.
\end{abstract}

Keywords: FIWARE $\cdot$ Agricolus $\cdot$ Smart Farming $\cdot$ Precision agriculture

\section{Introduction}

Recently, has been a growing interest in the use of new technologies, as Big Data and IoT, in areas with not many ICT influence such as agriculture. The application of technology represents an opportunity to improve current agricultural development, so new concepts like Smart Farming have been created to support agricultural production.

Moreover, research and innovation in this field is being encouraged from the EU Framework Program named Horizon 2020. The term Smart Farming is linked to concept Precision Agriculture and is viewed as a hardware infrastructure for precision agriculture. Precision Agriculture is a term introduced in the 1980 [1] and refers to the management of agricultural plot by monitoring, data processing and intervention of crops [2]. Besides, it contributes to optimize the consumption of resources, eradicates farm epidemic, and provides the farmers an added value of decision making for exploitation daily operations and management [3].

In agriculture industry has been developed some farm IoT systems, for example, a remotely controlled farm that can be managed using mobile devices [4] or a wireless automation system by monitoring and collect crop data [5]. 
In this paper, we focus on the farm IoT systems developed with technology FIWARE [6]. FIWARE is an open standard platform that belong Future Internet Private Public Partnership (FI-PPP) program. This program aims to advance Europe's competitiveness in digital technologies, and in particular, to support a new European Cloud platform [7].

A recent example of farm IoT system created with open infrastructure FIWARE is Agricolus. Agricolus has been launched by FIWARE Foundation and developed by TeamDev on March 2018. This product manages to bring Hardware and Software together in a decision-making process that support farming activities and offers a "plug and play" interface for precision agriculture [8].

Therefore, the main contributions of this paper are: the literature update of components of FIWARE platform, the proposal of an actual review of FIWARE in Smart Farming explaining previous studies and its most reused FIWARE components, a description of the new product Agricolus and its architecture decomposed in FIWARE components.

For that, literature search related to this topic is carried out within well-known databases, such as ScienceDirect, Elsevier and others. The remainder of this paper is organized as follows. In Sect. 2, a review of components of FIWARE platform and a FIWARE overview in Smart Farming are detailed. A description of the new platform for precision farming called Agricolus is described in Sect. 3. The detail of the new platform Agricolus in its components FIWARE is proposed in Sect. 4. Finally, in Sect. 5 conclusions of this paper are exposed.

\section{FIWARE in Smart Farming}

FIWARE is present in many different sectors in Europe, for example, healthcare, telecommunications, environmental services, and recently agriculture. The key of FIWARE is to be an open architecture and a reference implementation of a service infrastructure, building upon generic and reusable building blocks, available through Application Program Interfaces (APIs) called Generic Enablers (GEs).

\subsection{FIWARE Generic Enablers}

The GEs offer a number of general-purpose functions, easing development of smart applications in multiple sectors, and they are grouped into functional groups. Below, we describe the most relevant GE implementations of components catalogue FIWARE divided by groups. For each GE the FIWARE Foundation provides their specifications and implementations with an open source license, which is included in the catalogue [10].

(1) Services Ecosystem and Delivery Framework (SEDF): co-create, publish, crosssell and consume applications or services.

(i) Business API Ecosystem (called Biz Ecosystem RI): offers support for selling apps, data, and services. Version: 6.4.0. Updated: 2017-12-20.

(ii) Application Mashup (called Wirecloud): offers tools that allows users to create and run a web application front-end. Version: 6.4.2. Updated: 2017-11-01. 
(2) Cloud Hosting $(\mathrm{CH})$ : provides computation, storage and network resources to manage services.

(i) Application Management (called Murano): provides the basic support for hardware deployment management. Version: 5.4.3. Updated: 2016-11-03.

(3) Data/Context Management (DCM): easing access, gathering, processing, publication and analysis of context information at large scale.

(i) Cygnus: Cygnus is a connector in charge of persisting sources of data in certain configured third-party storages. Version: 4.2.1. Updated: 2017-05-16.

(ii) Publish/Subscribe Context Broker (called Orion): is an implementation of NGSI9 and NGIS10 with persistence storage. Version: - Updated: 201703-09.

(iii) BigData Analysis (called Cosmos): is intended to deploy means for analyzing both batch and stream data. Version: 1.0.0. Updated: 2016-12-12.

(iv) Complex Event Processing (called CEP): analyses event data in real time. Version: 5. Updated: 2016-08-07.

(4) Advanced middleware and interfaces to Networks and Devices (I2ND): build communication-efficient distributed applications, exploit advanced network capabilities and easily manage robotic devices.

(i) Kiara Advanced Middleware: is a Java based communication middleware for applications. Version: 0.4.0. Updated: 2016-02-04.

(5) IoT Services Enablement (IoTSE): make connected things available, searchable, accessible, and usable.

(i) Backend Device Management (called IDAS): connects IoT devices/gateways to FIWARE platform. Version: 7.1.3. Updated: 2018-02-12.

(ii) IoT Discovery: allows context producers to register their IoT Objects in linked-data format. Version: 5. Updated: 2016-10-26.

(iii) IoT Broker: is a middleware component enabling applications to retrieve information from IoT installations. Version: 6.4. Updated: 2018-01-17.

(iv) IoT Data Edge Consolidation GE (called Cepheus): provides a common access in real time to data, for sensors. Version: 1.0.0. Updated: 2017-06-15.

(6) Security (SEC): make delivery and usage of services trustworthy by meeting security and privacy requirements.

(i) Identity Management (called KeyRock): offers tools for administrators to support the handling of user functions. Version: 6.0.0. Updated: 2018-03-20.

(7) Advanced Web-based User Interface (AWUI): 3d \&AR capabilities for web-based UI.

(i) POI Data Provider: provides spatial search services and data on Points of Interest via RESTful. Version: 5.4. Updated: 2016-09-19.

When a software architect's develops a new agriculture solution or other kind of solution, it is not required to use all GE of the platform. The solution can use only the GEs that it demands for his case, because each GE of platform is independently. 


\subsection{FIWARE Generic Enables Used in Smart Farming}

In previous studies of Smart Farming, some studies present solutions for precision agriculture using IoT platform of FIWARE [11-17] IoT platform as \&Cube and Mobius [18] or ISOBlue [19]. In this paper, we focus in the studies based on used FIWARE:

Agri-IoT [11]: A semantic framework for IoT- based smart farming applications, which supports reasoning over various heterogeneous sensor data streams in real-time. Reused FIWARE GEs: IDAS and Cepheus.

SME Widhoc [12]: An application developed with the aim of reducing the amount of water necessary for irrigation tasks in real crops located in a semiarid area of the South of Spain. Reused FIWARE GEs: Orion, Cosmos, Cygnus and Wirecloud.

Testbed [13]: A testbed in a laboratory environment implemented to simulate different deployments and load conditions for analysis of an IoT use case scenario in the domain of precision agriculture. Reused FIWARE GEs: Orion, Cosmos, Cygnus, IoT Discovery and IoT Broker.

Cropinfra [14]: A comprehensive Internet-based networked crop production infrastructure to assist farmers to operate efficiently and fulfil farming demands using present and future technologies. Reused FIWARE GEs: CEP, Orion, Kiara Advanced Middleware, Business API Ecosystem, Wirecloud, IoT Broker and KeyRock.

FMS or Greenhouse [15-17]. A novel Business-to-Business collaboration platform from the agri-food sector perspective, which aims to facilitate the collaboration of numerous stakeholders belonging to associated business domains, in an effective and flexible manner. Reused FIWARE GEs: CEP, Orion, Kiara Advanced Middleware, Business API Ecosystem, Wirecloud, IoT Broker and KeyRock.

In these studies analysed, we can observe that Orion is the most reused FIWARE generic enablers, because it is reused in four out of five studies, followed by Wirecloud and IoT Broker that are reused in three out of five studies. Orion manage context information and is the mandatory component of FIWARE platform, Wirecloud displays data on dashboards and is essential for visualize context information, and IoT Broker is device interface and is key to retrieve information.

Other generic enablers such as Business API Ecosystem (seller of apps or services) or Kiara Advanced Middleware (communication middleware) are less reused because they aren't core generic enablers and are responsible of tasks that are outside the regular workflow of the context information (processing, analysis and visualization).

\section{Agricolus - A New Platform for Precision Farming}

In the agriculture-food sector, FIWARE Foundation has created an accelerator program called SmartAgriFood [20]. The SmartAgriFood accelerator is supporting Small and Medium-sized Enterprises (SMEs) developing smart services and mobile apps to be addressed in the agriculture-food sector. In particular, Agricolus, which has developed 
recently by TeamDev, is FIWARE IoT Ready certified and we can find in the official FIWARE Marketplace [21].

Also, TeamDev participates in other FIWARE programs like FIWARE iHubs network, being the provider of iHub Umbria (official iHub in Italy). The FIWARE iHubs programme supports local digital hubs to enrich their services, to build a network of tech-enabling communities and to encourage the formation of digital economy at a local level. iHub Umbria is based in building the community of local FIWARE developers and is the center dedicated to the adoption of FIWARE platform among local companies. This facilitates the creation of collaborative networks of developersto-developers, developers-to-local companies, local companies-to-local companies to be more competitive together at local level [22].

TeamDev as member of SmartAgriFood accelerator, created Agricolus with the main motivation of to obtain real-time information about crops and farms. With this information, farmers are helped in the difficult process of making decisions about their agricultural production that they are faced daily.

This solution is a cloud applications ecosystem for precision farming with multiple purposes: disease awareness and forecasts, crop monitoring, decision support system for treatments and fertilizers, farm management and end to end traceability bringing valuable information to final users. In addition, it focuses entirely on the European market and, in particular, it concentrates on small, medium and large farms. For the moment, as recently launched, their first customers are Italian farmers and farmers associations who grow wine, olives, vegetables, oat, corn, and tobacco [23].

An example of implementation to this project is shown on Fig. 1. It is the deployment in some tobacco's crops located in different geographical regions of Italy (Veneto, Umbria e Campania). The recollected data provided by sensors installed in tobacco's crops are used to support decisions about agricultural operations like sowing
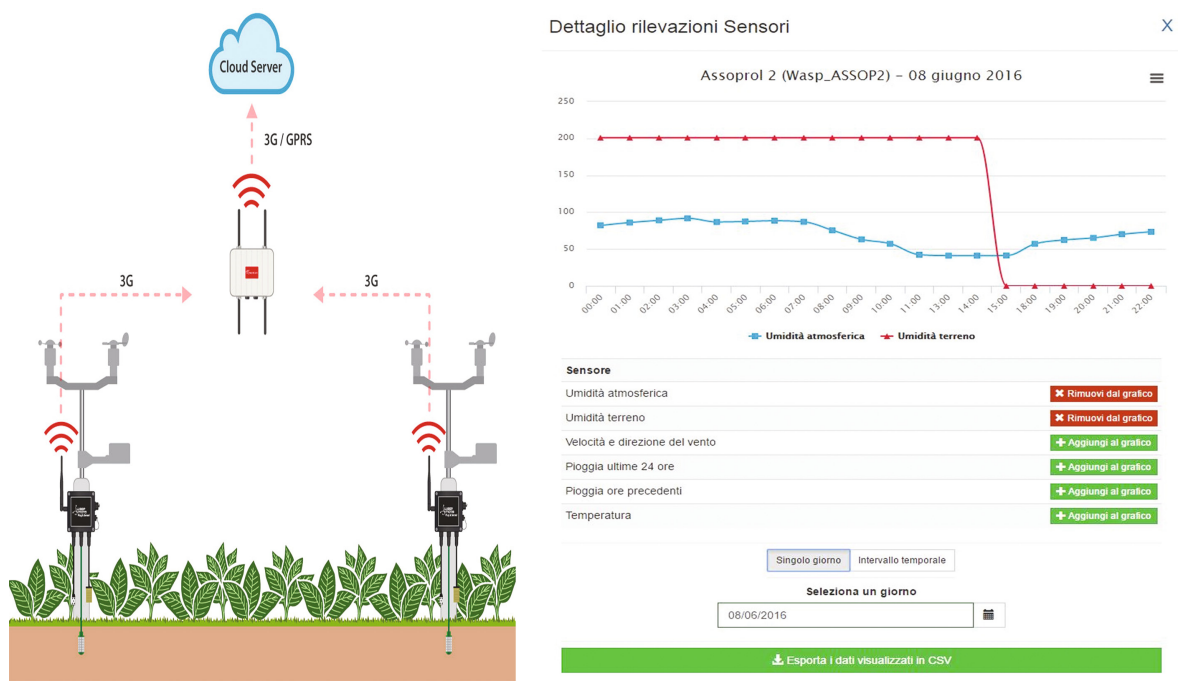

Fig. 1. Implementation example of Agricolus in tobacco's crops in Italy. 
or harvest time, timing for treatments and predictive models to spread pests. All data converge in Agricolus suite for managing tobacco's crops and extract insight can guide cultivation techniques, essential to improve tobacco's quality [24].

Besides, Agricolus offers a Web application, which allows farmers to map fields combining external GIS (geographic information system) services and analyses information from crop management which stores in Agricolus platform. The web application shows a map with different types of geolocated crop and displays information about these crops (such as vegetation indices calculated or weather indicators) [8].

Likewise, to improve indicators information which shows on his Web application, Agricolus has integrate FIspace (another project that belongs to FI-PPP program) with his services. FIspace is a business-to-business (B2B) collaboration platform and aims at developing solutions to address the challenges in collaborative business networks, focusing on use cases from the agri-food or transport industries. It works like a social network, like LinkedIn or Facebook [25].

With FIspace the company can negotiate collaborations, manage intercompany business processes, exchange data or deliver and use value-added services; this is quite important to small or medium sized companies without enough technological resources. For instance, it only stores the links between business and the conditions for data sharing (never store the business data exchanged), with this programm will facilitate seamless B2B Collaboration, efficient developments of customized solutions with minimal costs and evolution of open business networks [25].

\section{Components of Agricolus, a New FIWARE Architecture Use Case}

In this paper, the main goal is to present systems developed with FIWARE technology in the agriculture area and compare which FIWARE components (generic enablers in this case) are reused on these systems; for store, analyze and share agricultural data by IoT devices in connected farms, designed with the purpose of monitoring and controlling daily tasks in real farms or real agricultural environments. For that, we describe the FIWARE Generic Enables used in Agricolus.

The Agricolus solution centers on the following process: (1) collecting data from several sources such as sensors and devices, (2) organizing and analyzing the collected data to create historical data, (3) utilizing this data to compose the application.

Agricolus uses FIWARE to the first and second step of the process (collect data from the sensors and bring them together to be analyzed and stored), which reuse FIWARE GEs as Orion that manage the entire lifecycle of context information including updates, queries, registrations and subscriptions; IDAS (specifically IoTAgent - UltraLight2.0 protocol) which sends data from sensors to Orion (registrations and updates); Cosmos that stores and analyzes data; Cygnus which is responsible of the data transport between Orion and Cosmos and KeyRock that offers identity management to configure private authentication from sensors and devices.

The Table 1 shows the number of FIWARE generic enablers reused in the studies analysed in Sect. 2.2, and the new Agricolus solution, grouped by the categories mentioned in Sect. 2.1. According to the table it can be noticed that DCM group is the 
most reused, because it is essential the data/context management for the IoT platform, for example Orion, Cygnus and Cosmos are core generic enablers in standard architecture FIWARE. Moreover, Orion is the star generic enablers, because it is reused in five out of six solutions and it seems essential for any IoT platform in Smart Farming.

Table 1. Number of FIWARE generic enablers reused in each group.

\begin{tabular}{l|l|l|l|l|l|l|l}
\hline Smart Farm solution & SEDF & CH & DCM & I2ND & IoTSE & SEC & AWUI \\
\hline Agri-IoT & 0 & 0 & 0 & 0 & 2 & 0 & 0 \\
\hline SME Widhoc & 1 & 0 & 3 & 0 & 0 & 0 & 0 \\
\hline Testbed & 0 & 0 & 3 & 0 & 2 & 0 & 0 \\
\hline Cropinfra & 2 & 0 & 2 & 1 & 1 & 1 & 0 \\
\hline FMS or Greenhouse & 2 & 0 & 2 & 1 & 1 & 1 & 0 \\
\hline Total present applications & 5 & 0 & 10 & 2 & 6 & 2 & 0 \\
\hline Agricolus & 0 & 0 & 3 & 0 & 1 & 1 & 0 \\
\hline Total with Agricolus & $\mathbf{5}$ & $\mathbf{0}$ & $\mathbf{1 3}$ & $\mathbf{2}$ & $\mathbf{7}$ & $\mathbf{3}$ & $\mathbf{0}$ \\
\hline
\end{tabular}

\section{Conclusions}

This paper has identified the existing solutions in Smart Farming used by FIWARE and has compared FIWARE components (generic enablers) reused on these systems. Also, we have updated literature review of components of FIWARE platform and we have described a new platform for precision farming called Agricolus.

These solutions studied provide tools accessible to worldwide farmers and consumers too. These are good news for the agricultural sector, which can adopt these open technologies in their farms and create connected farms. By combining the FIWARE platform with other third-party developers as ArcGis (cartographic representation tools) that allowing the crop data to be visualized in maps in order to understand patterns and trends which improve the management farm.

To conclude, we observed that Publish/Subscribe Context Broker (implementation called Orion) belonging to DCM group, is the most generic enabler reused in solutions analyzed in Smart Farming, so data/context management can seem essential for any Smart Farming IoT platform. Therefore, FIWARE is a powerful open platform and it is a useful way to standardize the adoption of common interfaces in the IoT field in many different sectors in Europe as agriculture.

Acknowledgments. This research has been carried out in the framework of the project "Development of an integrated maturity model for agility, resilience and gender perspective in supply chains (MoMARGE). Application to the agricultural sector.” Ref. GV/2017/025 funded by the Generalitat Valenciana. 


\section{References}

1. Robert, P.C.: Precision agriculture: research needs and status in the USA. In: Stafford, J.V. (ed.) Proceedings of the 2nd European Conference on Precision Agriculture, Part 1, pp. 1933. Academic Press, SCI/Sheffield (1999)

2. Ge, Y., Thomasson, J.A., Sui, R.: Remote sensing of soil properties in precision agriculture: a review. Front. Earth Sci. 5(3), 229-238 (2011)

3. Sundmaeker, H., Verdouw, C., Wolfert, S., Pérez Freire L.: Internet of food and farm 2020. In: Vermesan, O., Friess, P. (eds.) Digitising the Industry - Internet of Things Connecting Physical, Digital and Virtual Worlds, pp. 129-151. River Publishers, Gistrup/Delft (2016)

4. Lin, J., Liu, C.: Monitoring system based on wireless sensor network and a SocC platform in precision agriculture. In: Proceedings of the International Conference on Communication Technology (ICCT), Hangzhou, pp. 101-104 (2008)

5. Kaewmard, N., Saiyod, S.: Sensor data collection and irrigation control on vegetable crop using smart phone and wireless sensor networks for smart farm. In: Proceedings of the International Conference on Wireless Sensors (ICWiSE), pp. 106-112 (2014)

6. FIWARE. https://www.fiware.org/

7. Future Internet Private Public Partnership (FI-PPP). https://www.fi-ppp.eu/

8. Agricolus. https://www.agricolus.com

9. FIWARE Generic Enablers. http://edu.fiware.org/

10. FIWARE Catalogue. https://catalogue.fiware.org/enablers

11. Kamilaris, A., Gao, F., Prenafeta-Boldu, F.X., Ali, M.I.: Agri-IoT: a semantic framework for Internet of Things-enabled smart farming applications. In: IEEE 3rd World Forum on Internet of Things, WF-IoT 2016, pp. 442-447 (2017)

12. López-Riquelme, J.A., Pavón-Pulido, N., Navarro-Hellín, H., Soto-Valles, F., TorresSánchez, R.: A software architecture based on FIWARE cloud for precision agriculture. Agric. Water Manag. 183, 123-135 (2017)

13. Martínez, R., Pastor, J.Á., Álvarez, B., Iborra, A.: A testbed to evaluate the FIWARE-based IoT platform in the domain of precision agriculture. Sensors (Switzerland), 16(11) (2016)

14. Pesonen, L.A., et al.: Cropinfra - an internet-based service infrastructure to support crop production in future farms. Biosys. Eng. 120, 92-101 (2014)

15. Barmpounakis, S., et al.: Management and control applications in agriculture domain via a future internet business-to-business platform. Inf. Process. Agric. 2(1), 51-63 (2015)

16. Kaloxylos, A., et al.: Farm management systems and the future internet era. Comput. Electron. Agric. 89, 130-144 (2012)

17. Kaloxylos, A., et al.: A cloud-based farm management system: architecture and implementation. Comput. Electron. Agric. 100, 168-179 (2014)

18. Ryu, M., Yun, J., Miao, T., Ahn, I.Y., Choi, S.C., Kim, J.: Design and implementation of a connected farm for smart farming system. In: 2015 IEEE SENSORS Proceedings, pp. 1-4 (2015)

19. Layton, A.W., Balmos, A.D., Sabpisal, S., Ault, A., Krogmeier, J.V., Buckmaster, D.: ISOBlue: an open source project to bring agricultural machinery data into the cloud, Montreal, 13 July-16 July 2014. American Society of Agricultural and Biological Engineers (2014)

20. SmartAgriFood. http://smartagrifood.com/

21. FIWARE MarketPlace. https://marketplace.fiware.org

22. FIWARE iHubs. https://www.fiware.org/community/fiware-ihubs/ 
23. Agricolus in FIWARE MarketPlace. https://marketplace.fiware.org/pages/solutions/2ec3c 741ef4dd8f83bab4e83

24. Implementation example of Agricolus. http://www.libelium.com/increasing-tobacco-cropsquality-by-climatic-conditions-control/

25. FIspace. https://www.fispace.eu/whatisfispace.html 